\title{
Problems and Directions of Development through Analysis of Virtual Reality-Based Education in Korea
}

\author{
Mi Young An, Kyung A Ko, and Eun Ju Kang
}

\begin{abstract}
The purpose of this study is to examine what kinds of virtual reality-based education have been in Korea and what positive effects it has on learners. In addition, this study examines the limitations of current virtual reality-based education and the directions to be developed. For this purpose, the research was conducted through the literature review method that finds and organizes the documents which are the data of this study, such as various papers, research reports and articles. As a result of this study, the virtual reality-based education has a more realistic experience through the development of virtual reality technology, free movement, physical and sensory interactions. Many companies are growing quantitatively and qualitatively by introducing into various services and contents fields such as education, medical, film, military, sports and tourism in anticipation that the development of network and virtual devices will be greatly improved in the future. In particular, many attempts have been made in education, medicine and sports. Virtual reality-based education provides learners with a three-dimensional and realistic environment to deepen and broaden the learning experience. The learner is immersed in learning and gains practical experience, and the learner becomes the self-directed learner who can achieve the output. The limitations of virtual reality include cyber sickness, low vision and lack of content.
\end{abstract}

Index Terms-Virtual reality, virtual reality based education, effect of virtual reality based education.

\section{INTRODUCTION}

Recently, the mobile population has rapidly increased, and mobile contents are being developed and used throughout society, economy, and culture [1]. Virtual reality (VR), one of the core areas of the Fourth Industrial Revolution, is an important technology that will change our lives in the future, and as a result of the increasing interest of many companies, it will be expanded to various industries such as safety, defense, medicine, games, and education [2].

Virtual reality is a technology that creates a virtual environment, rather than a reality, through a graphic, and allows the user to experience the virtual world through virtual reality. In a dictionary, it is an interface between a human and a computer that makes a particular environment or situation into a computer and makes the user use it as if he or she is interacting with the surrounding environment [3]. In addition to the commercialization of $5 \mathrm{G}$, the next generation network, VR is expected to increase the popularity of realistic content. Virtual reality technology is most utilized in entertainment

Manuscript received March 19, 2020; revised June 3, 2020.

Mi-Young An, Kyung-A Ko, and Eun-Ju Kang are with Department of Early Childhood Education, Cheju Halla University, South Korea (e-mail: myahn.ange@gmail.com, dmswn5788@naver.com). fields such as games and movies. In the field of education, it is used in learning contents and abstract learning concepts that are difficult to directly observe in the situation or environment or that are difficult to explain in writing or video materials. In addition, it is used as a means of building a learning environment to increase learning effects by applying to dangerous or expensive experiments.

Educational contents using virtual reality are more effective than other educational contents in terms of reality and immersion. Education through virtual reality enables experimental and active participation, promotes cooperation and motivation for learning with other learners, and enables learning through virtual reality that is impossible in reality [4].

The purpose of this paper is as follows. First, let's take a look at Korea's use of virtual reality. Second, this study examines whether virtual reality-based education in Korea has a positive effect on learners. Third, suggest what are the limitations of the present and the directions for future development.

\section{MAIN SUBJECT DEFINITION AND BACKGROUND OF VIRTUAL REALITY (VR)}

VR stands for Virtual Reality and refers to a technology that allows users to perceive and immerse themselves in reality by constructing a virtual space using a computer [5]. In simple terms, it refers to the interface between humans and computers that creates a special environment, a situation using a computer, and makes it possible for people who use it to feel and interact with reality. VR, which realizes virtual environment with 3D virtual background created through computer, can be easily immersed in user's environment, so emphasize the importance of 'user experience', 'immersion' and 'reality' in VR. In other words, the core of VR technology is an immersive environment where users of VR can concentrate and a natural background without any sense of heterogeneity. VR creates a natural virtual world through Head Mounted Device (HMD), a monitor that wears like glasses, providing high immersion to the user [3].

In the background of the emergence of VR, the term VR first began in 1938 when French playwright Antoine Arto described the theater as a 'virtual reality space' that focused the audience. In 1965's Ivan Sutherland's speech, he said: "In the future, the objects we will create with computers will be so real that the viewer will not be able to distinguish between the virtual objects and the real things created by computers." HMD for virtual reality was developed, and the possibility of this was known when Jaron Lanier first commercialized virtual reality technology based on HMD in 1985 [6], [7]. 
However, with the high price that the public cannot buy, the technology is gradually forgotten. Due to the high price that the public cannot buy, the technology was soon forgotten. After that, in 1989, Jaron Lanier began to use the word 'virtual reality' and became the first to make VR-related products and lead the popularization of VR. VR, which became increasingly popular around the world, reached a long downturn over time. It was the product of a company called Oculus that was acquired by Facebook in this downturn. Oculus was convinced of the possibility of VR and again led the popularization of VR, which became the predecessor of the current VR HMD [8].

\section{VIRTUAL REALITY-BASED EDUCATION USAGE}

\section{A. Review Stage Educational Use of Virtual Reality}

In the early days, virtual reality was used to visualize information by focusing only on information transmission. However, due to technical limitations at that time, there were limitations due to low immersion and realism. With the development of current technology, free movement and physical and sensory interactions have been made possible from the information transmission means [9]. Experiences or education through virtual reality have been introduced by many companies in the expectation that the educational effect will be shown, as well as the development of networks and virtual devices will be greatly improved in the future. Recently, various services and contents fields such as education, medical, film, military, sports, tourism are growing quantitatively and qualitatively. In particular, many attempts have been made in education, medicine and sports.

In Korea, education is being conducted in various areas based on virtual reality. First, let's look into the flow and direction of domestic virtual reality-based education. Beginning in 1995, Korea's first virtual reality-based learning simulation education program called 'YM VR ANATOMY' is used for medical school and nursing school students, for anatomical support, doctors and Chinese doctors, and for clinical use [10].

In 2000, the virtual reality-based " Smart Class" experience classroom system was designed to make people feel as real as they see when teaching the natural sciences such as biological earth sciences, celestial bodies, and physics as well as the humanities [11]. In 2003, a virtual experience solution for architecture education was developed with the ability to look at more diverse locations, providing the ability to change the internal design and then simulate it [12]. In addition, vocational job training and safety education based on virtual reality are being conducted [13].

Looking at the virtual reality-based education of the 2000s, it can be seen that the subject mainly consists of a simulation education that acquires knowledge or develops coping ability by experiencing it like a real situation in a situation that is not actually experienced through virtual reality-based education in Korea. In 2005, the education office of Incheon city provided virtual reality experience learning for elementary, middle and high school students. We experienced culture, traditional houses and museums of each country in virtual reality [14].
In addition, in the same year, 'winter vacation experience class' was opened for elementary school students. The program consists of hands-on education to experience and make traditional Korean style house based on virtual reality [15]. It was the first domestic library to offer a storytelling service based on virtual reality. From the late 2000s, we can see that the subjects of virtual reality-based education are elementary, middle, and high school, and the subjects of various ages are created and various experiences are realized in the virtual reality.

In the case of education using virtual reality technology, the Ministry of Education based the virtual reality on realistic digital textbook. The Ministry of Education produces realistic content in digital textbooks in 2018 and actually implements them in the curriculum, attracting good responses from learners, expanding the grade targets, and promoting further development projects. In addition, safety education, a curriculum, is being expanded into a education project through virtual reality experience. Since 2019, it has been offering realistic content classes based on virtual reality. The Ministry of Education said it will develop virtual reality content for the social and scientific digital textbooks and art and mathematical content. It is also spreading HMD devices in digital textbook research schools to test educational effects based on virtual reality and to lead the development of educational contents based on virtual reality. Secondly the health care virtual reality content is provided as a nursing college virtual reality.

This virtual reality content is composed of adult nursing practice and anatomy practice. In the practice of anatomy, it is possible to learn the function of the human body structure and the three-dimensional and specific shape through the virtual reality contents, and to see the mutated state of the organ according to the disease as in the actual field [1].

Industry also needs virtual reality-based training. Simulations combined with hands-on virtual factory environments can be used to create a familiar virtual environment, training workers and enhancing work proficiency in a realistic environment, with virtual spaces that are exactly the same as the site.

In addition, they can raise awareness about disasters, raise safety awareness, and improve their ability to cope with actual accidents. On the other hand, the site is also conducting stability experiences on equipment such as hard hats, safety impacts, and ladder conduction experiences [1], [16].

In the military and police education and training sectors, virtual reality-based education and training simulations are better used than others. Traditional military exercises have been used to train soldiers by simulating combat situations. This is less realistic and expensive, so the training effect is not high. In addition, in the case of police training, it is difficult to provide effective training because it is not easy to reproduce the crime situation.

Therefore, VR simulation is attracting attention as an alternative to resolve these problems, and it reproduces the simulated combat situation and crime situation in the virtual environment, enabling the trainer to train the necessary skills and behavioral tips [17]. 
TABLE I: VIRTUAL REALITY EDUCATION BY YEAR

\begin{tabular}{|c|c|c|c|}
\hline & 1995-early 2000s & Mid 2000s & $2010 \sim$ \\
\hline Characteristic & $\begin{array}{l}\text { - University and Adult Learner Focused } \\
\uparrow \\
\text {. Simulation education }\end{array}$ & \multicolumn{2}{|c|}{$\begin{array}{c}\text { Expanded to elementary, middle and high schools for virtual } \\
\text { reality-based education } \\
\cdot \text { Various experiences available }\end{array}$} \\
\hline $\begin{array}{l}\text { VR } \\
\text { education-based } \\
\text { content }\end{array}$ & $\begin{array}{c}\text { <Learner Program for Children> } \\
\text { (1) 'Smart class': 3D Experience Class System Natural } \\
\text { Sciences and Humanities (2000) }\end{array}$ & $\begin{array}{l}\text { <Learner Program for Children> } \\
\text { (1) Virtual reality experience learning: } \\
\text { Experience culture in other countries, } \\
\text { Voice recognition English learning service - } \\
\text { Voice recording and pronunciation } \\
\text { comparison(2005) } \\
\text { (2) Elementary school student 'Winter } \\
\text { vacation experience learning class': } \\
\text { Traditional Korean Style House Experience } \\
\text { and Making Experience Education (2005) } \\
\text { (3) 3D storytelling service: Interaction based } \\
\text { virtual experience - the first program in a } \\
\text { domestic library (2009) }\end{array}$ & $\begin{array}{l}\text { (1) Realistic Digital } \\
\text { Textbook(2018): } \\
\text { Virtual reality-based } \\
\text { realistic content } \\
\text { classes starting from } \\
2019 \\
\text { (2) Health care virtual } \\
\text { reality contents } \\
\text { (2018): Long-term } \\
\text { variation according to } \\
\text { human structure and } \\
\text { disease } \\
\text { (3) Industry: } \\
\text { roficiency } \uparrow, \text { Raising } \\
\text { awareness about } \\
\text { disasters and raising } \\
\text { safety awareness } \\
\text { (2018) } \\
\text { (4) Training field: } \\
\text { Military and police } \\
\text { training with virtual } \\
\text { simulation } \\
\text { (2018) }\end{array}$ \\
\hline
\end{tabular}

\section{B. Virtual Reality Implementation Method and Technology Element}

There are important factors for the implementation of virtual reality (VR). First of all, users need to create sensory experiences that look as if they exist in the place through the five senses such as vision and hearing. The three-dimensional space created by technicians is a virtual space, making it as close to reality as possible, or being created as if it does not exist but as if it exists [18]. In order to create a sensory experience, we must create a virtual reality that is disconnected from the real world and provide an indirect experience that is like being in the real world. This is called virtual presence and is one of the important elements of VR. According to the results of scholars' research, there are various factors that influence the formation of virtual reality, and the formation of virtual reality is the main factor for implementing virtual reality [19].

In order for the learner to think as if he is in the space, the output device should be a device that controls the vision to immerse the view [18]. Virtual reality devices include controllers, VR headsets, haptic equipment, materials and components, and gloves [20]. Among them, a representative virtual reality device is a head-mounted display (HMD), which is a head-mounted device worn on the head, and uses it to immerse deeper into the world of virtual reality [21]. Lastly, real-time interaction shows the response in real-time through immediate input and processing of various reactions of learners, and minimizes the difference between reality [18] Virtual reality makes you feel artificial reality by realizing a fake environment that does not exist as a reality. This allows the learner's interaction to be immersed in a life-like experience [22].

\section{Advantages and Effects of Virtual Reality-Based Education}

Virtual reality-based education, learners actively manipulate objects. In addition, he said that learners are immersed in learning, gaining practical experience, and learners become a main character who tries to lead learning. Virtual reality-based education is said to have a greater effect on academic achievement than classical education that injects existing reading and listening knowledge [2]. According to one study, virtual reality-based education typically has a 2.7-fold increase in effectiveness and concentration by over $100 \%$ compared to conventional education [23]. It has a positive effect on the effect. Thus, the positive result of the improvement of academic achievement through the interest and immersion of learning is shown. In addition, wearing HMD removes the obstacles to pay attention, so it concentrates on more tasks. Distracted learners through virtual reality-based education also showed that attention concentration was maintained [24].

\section{Problems and Limitations of Virtual Reality-Based Education}

Virtual reality has many problems to solve for learners. Typical examples include cyber sickness (dizziness), space limitation, difficulty in popularization due to cost, difficulty in distinguishing between virtual and reality, and limitation of dynamics of the real world [25]. Children can wear dizziness for a long time so that dizziness can be observed when children start to go beyond 10 minutes [26]. In addition, exposure to the screen for a long time at a narrow distance may affect children who are still developing the optic nerve. The younger you need to be more careful in using virtual reality. Long-term wear causes photosensitivity seizures, in other words called Pokemon seizures, or Nintendo seizures. Care should be taken in the use of virtual reality, which is visually stimulating as it happens when more light is exposed than necessary. In addition, the scope of use will expand as virtual reality is diversified into various areas. To this end, studies of stability in a wide variety of areas are needed to minimize the various risks that may be present. 
Virtual reality education is inevitably opposed to traditional education, and it is not appropriate for the education of law and system, so education in humanities will be limited [23]. Another reason why the field of virtual reality education is not successful is the lack of killer content and lack of content. In particular, the education market is urgently needed to develop contents that can be synergistic with the existing system. Virtual reality learners are interested in game contents that can be interesting and fun and tend to be preferred. Even if applied to other fields, it can be said that in the form of content development, it is efficient in terms of popularization and activation of virtual reality-based programs as well as learning effects. Therefore, there is a necessity to develop various game-type educational contents so that learners can have fun and interest in the effective aspect of learning [2].

\section{E. Direction of Virtual Reality-Based Education}

The core values provided by virtual reality will provide a different environment for learners to realize themselves. The emergence of the $5 \mathrm{G}$ era will create demand for new services that have not existed before, and devices, and content will evolve from the present time, and will not only eliminate speed lags or interruptions by simply improving speed, but also become more realistic [23], [27]. Virtual reality program has the advantage of being able to experience real virtual space without limit through relatively low cost and high immersion. However, due to lack of technology and low resolution, there is a technical limitation that can cause some motion sickness caused by cyber sickness. In addition, there is a problem that the experience in a narrow and inconvenient space can act as a risk factor to the user due to the wearing method of HMD, and a lot of research is being done to resolve these problems [28]. First, in terms of the constraints of Daum Space, contents that can walk in a relatively wide space called 'Walking Attraction' are currently appearing, and technology is being developed to remove the space limitation [2]. In addition, it can cause mental problems that can confuse real and virtual reality, and it can cause vomiting and dizziness. Therefore, it is necessary to study with other fields such as medicine and psychology [29]. Virtual reality is currently evolving into a technology that allows users to communicate with each other, such as being in different spaces but in the same virtual space on a single device that is disconnected from the outside by wearing HMD. It also scans the movements of users in different spaces and sends them to a virtual environment to provide an experience for interacting with various people [30]. One news article cited a negative stance as the biggest problem in the application of the new technology of virtual reality in education. The use of virtual reality requires a period of skill testing and adaptation to new technologies, and both students and teachers must go through intensive training to learn and learn virtual reality. For this purpose, not only the basic learning about virtual reality but also the necessity of the virtual reality education law, which is the foundation for teachers to lead in-depth classes, is raised [31], [2]. Because of the wide range of possible effects, detailed and broad user research should be conducted from various perspectives, including medicine and sociology. Through this, it is necessary to minimize the side effects that can occur and overcome the technical limitations [30].

\section{CONCLUSION AND SUGGESTIONS}

In the past, only limited-quality content in 3D TV could be viewed in $3 \mathrm{D}$, but now, in the era of virtual reality, we are seeing the $5 \mathrm{G}$ revolution. This may not be enough for VR killer content right now, but now the era of cloud is opening up, where you can enjoy high-quality game services on low-performance hardware devices [32]. Virtual reality education can be said to have a high possibility of development because it can be moved to distance learning or an independent learning content in that actual activity takes place in a more realistic situation. Accordingly, there is a need to actively attempt to use new tools and technical characteristics in various educational contexts [33].

In this study, I would like to suggest the direction of the virtual reality education in the future by analyzing through the thesis on the virtual reality based education in Korea.

First, when wearing HMD for a long time, it is more dangerous for young children who are growing their bodies. In the future, virtual reality will expand to a wider range as its use becomes more diverse. The problem of virtual reality in this situation causes difficulty for learners when applying educational contents. Active research to minimize this should be done to ensure the correct use of the device and to minimize side effects.

Secondly, as times has been changed, the supply of HMD devices to virtual reality is increasing due to the development in IT industry. In this situation, many companies are expanding to content competition instead of device supply. Interesting content has the advantage of improving learners' concentration, immersion, and academic achievement. Therefore, many companies need to develop contents with high interest and education in virtual reality education contents.

Third, virtual reality is now easily seen around us. Children of younger age can also enjoy the small screen of smartphones and HMD devices easily through various contents Many users, as well as young children, have easy access to a variety of corporate-developed content because of their curiosity and interest, and indiscriminate exposure to them presents risks. Accordingly, age-based content should be restricted, and content suitable for the target should be produced and recommended for use.

\section{CONFLICT OF INTEREST}

The authors declare no conflict of interest.

\section{AUTHOR CONTRIBUTIONS}

An conducted the research; Ko and Kang analyzed the data; Ko and An wrote the paper, all authors had approved the final version.

\section{REFERENCES}

[1] K. H. Park, "Educational application of virtual reality technology," Korea Robotics Society Review, vol. 15, no. 4, pp. 28-36, 2018.

[2] J. H. Lee, "A study on the revitalization of virtual reality-based education," Journal of the Korean Society Design Culture, vol. 25, no. 1, pp. 357-366, 2019. 
[3] J. S. Lee and J. Y. Kim, "A study on spatial characteristics of immersion and reality in cases of VR and AR technology and contents," Journal of the Korean Institute of Interior Design, vol. 28, no. 3, pp. 13-24, 2019.

[4] S. M. Sung and M. G. See, "A study of trend and effectiveness of imprinting effect in VR, AR educational contents," in Proc. KSDS Conference, 2017, pp. 208-209.

[5] M. H. Yoo, J. H. Kim, Y. H. Koo, and J. H. Song, "A meta-analysis on effects of VR, AR, MR-based learning in Korea," Journal of Korean Association for Educational Information and Media, vol. 24, no. 3, pp. 459-488, 2018.

[6] J. S. Oh, H. C. Youn, H. J. Jeon, and S. M. Ko, "The thought on the new recognition of a virtual reality," in Proc. Korea Contents Association Conference, 2019, pp. 171-172

[7] H. S. Choi, "New evolution of virtual reality and social change," Future Horizon, vol. 29, pp. 15-19, 2016.

[8] I. H. Cho, "AR / VR present and future and SKT's R \& D strategy," Korean Journal of Multimedia, vol. 21, no. 12, pp. 23-31, 2017.

[9] J. H. Kim, "Industrial impact and implications of virtual reality technology," Future Horizon, vol. 29, pp. 8-11, 2016.

[10] Human anatomy learning product in 'New SW Product'. (1995). Yonhap News Agency. [Online]. Available: https://news.naver.com/main/read.nhn?mode=LSD\&mid=sec\&sid1=1 $05 \&$ oid $=001$ \&aid $=0003933722$

[11] C. G. Lee. (December 2000). 3D experience classroom system development. Hankyung. [Online]. Available: https://news.naver.com/main/read.nhn?mode=LSD\&mid=sec\&sid1=1 01 \&oid $=015$ \&aid $=0000316925$

[12] G. T. Park. (January 2003). Development of a virtual experience solution for high tech media and architecture classes. Etnews. [Online]. Available:

https://news.naver.com/main/read.nhn?mode=LSD\&mid=sec\&sid $1=1$ $05 \&$ oid $=030$ \&aid $=0000023642$

[13] J. H. Jeoung. (September 2003). POSCO, opened the 3D safety accident experience center. Etnews. [Online]. Available: https://news.naver.com/main/read.nhn?mode=LSD\&mid=sec\&sid $1=1$ $05 \&$ oid $=030$ \&aid $=0000043042$

[14] S. W. Moon. (August 2005). Classes to copy and look at blackboards are old words. Munhwa. [Online]. Available: https://news.naver.com/main/read.nhn?mode=LSD\&mid=sec\&sid1=1 02\&oid=021\&aid=0000117982

[15] H. J. Yoon. (December 2005). Opened winter vacation experience learning class for elementary school students at IT World. Inews24. [Online].

Available: https://news.naver.com/main/read.nhn?mode=LSD\&mid=sec\&sid1=1 05\&oid $=031$ \&aid $=0000074469$

[16] H. N. Kim. (July 2019). Prevent risks from construction sites and respond to them in advance. Korean Economy. [Online]. Available: https://news.naver.com/main/read.nhn?mode=LSD\&mid=sec\&sid1=1 01\&oid=015\&aid $=0004170300$

[17] H. R. Jeong, "Training simulation based on virtual reality," in Proc. Spring Conference of the Korean Society of Mechanical Engineers, 2018, pp. 217-218.

[18] H. G. Kim, Y. J. Son, M. S. Kim, and S. J. Lee, “Ambiguous boundary between reality and virtual reality and future of AR (augmented reality) vs VR (virtual reality) vs MR (mixed reality)," Defense \& Technology, vol. 455, pp. 76-87, 2017.

[19] J. H. Ryu and S. B. Yoo, "The effects of head mounted display on time-on-task and virtual presence for navigational tasks in the immersive virtual reality," Science of Emotion \& Sensibility(KOSES), vol. 19, no. 3, pp. 71-80, 2016.

[20] Y. C. Jang, J. S. Lim, and J. H. Kim, "Virtual augmented reality (VR / AR) industry trends," The Journal of The Korean Institute of Communication Sciences, vol. 36, no. 1, pp. 35-41, 2018.

[21] B. Y. Jeong, "Virtual Reality (VR) ecosystem status and implications," Information Communication Broadcasting Policy, vol. 28, no. 7, p. 621.

[22] S. Y. Jang, Develop and Apply Virtual Reality Education and Training Content, 2018.

[23] J. H. Choi, "The future of education and culture industry through virtual reality," Future Horizon, vol. 29, pp. 20-23, 2016.

[24] S. J. Kim, T. Y. Kim, and C. S. Lim, "Mixed-reality based situation training system for the developmental disabled," Journal of the Korea Computer Graphics Society, vol. 14, no. 2, pp. 1-8, 2008.

[25] J. H. Lee, "The flow and prospect of virtual reality in the 4th industrial revolution era," Media \& Education, vol. 7, no. 1, pp. 41-53, 2017.
[26] M. You, "Haptic VR contents of art education for children: Focused on "Hephys" VR," The Korean Journal of Animation, vol. 13, no. 3, pp 62-79, 2017.

[27] J. H. Park, "Get ready for VR evolving to next media," Industrial Engineering Magazine, vol. 26, no. 1, pp. 37-40, 2019.

[28] E. J. Song, "Virtual reality industry analysis and how to activate," Journal of the Korea Institute of Information and Communication Engineering, vol. 22, no. 4, pp. 656-663, 2018

[29] T. W. Han, "A study on the development direction of virtual reality games," The Society of Korea Illusart, 2017.

[30] B. S. Yang, "Virtual reality / augmented reality technology development direction and implications," SPRI, 2017.

[31] H. G. An. (October 2019). Why is AR / VR so difficult to spread in the education market? Inews24. [Online]. Available: https://news.naver.com/main/read.nhn?mode=LSD\&mid=sec\&sid $1=1$ 05\&oid $=031$ \&aid $=0000512749$

[32] J. H. Choi. (October 2019). Virtual reality never dies. Economic Reviews. [Online]. Available: http://www.econovill.com/news/articleView.html?idxno=375741

[33] C. I. Lim, "Redirecting the research and practice of educational technology for future society and education," Journal of Educational Technology(JET), vol. 35, no. 2, pp. 253-287, 2019

Copyright $\odot 2020$ by the authors. This is an open access article distributed under the Creative Commons Attribution License which permits unrestricted use, distribution, and reproduction in any medium, provided the original work is properly cited (CC BY 4.0).

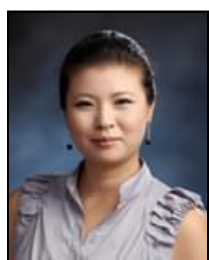

Mi-Young An was born in Incheon, South Korea in 1971. She received her bachelor of education in 2003 from Salesian Pontifical University, Rome, and her master of education administration and doctor of education in sociology of education from Salesian Pontifical University, Rome, Italy in 2004 and 2010, respectively. She is an associate professor in the Department of Early Childhood Education at Cheju Halla University, Jeju Special Self-Governing Province, South Korea. She has a wide range of research interests, which include futures thinking, educational change, globalization, teaching methods and early childhood education. Her research interests in recent years focus on the integration of futures and design thinking — particularly in engineering education. Prof. An is a current member of Korean Educational Research Association, the Korean Society for Sociology of Education, the Korean Association of General Education, and the Korean Association for Learner-centered Curriculum and Instruction. She is also active members of Korea Academy-Industrial Cooperation Society, Digital Contents Society, and Korean Information Electron Communication Technology.

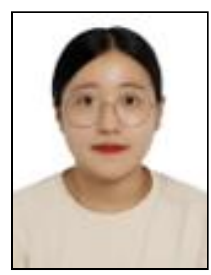

Kyoung A Ko was born in Jeju, South Korea in 1998 She received the professional bachelor of the early childhood education in February 2019 from Cheju Halla University, Jeju, South Korea and she has acquired the $2^{\circ}$ kindergarten instructor from Ministry of Science, Education and Technology and the $2^{\circ}$ childcare provider from Ministry of Families and Social Welfare. She will be received the bachelor of the early childhood education in February 2020 from Cheju Halla University, Jeju, South Korea. She has been working at the Jeju Island Cheong Nursery since March 4, 2019. Currently, she is interested in the prospect of future education and wants to study further development for today's education and how education will emerge in the future

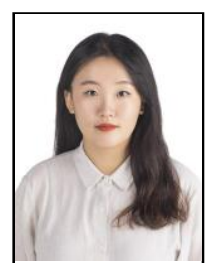

Eun-Ju Kang was born in Jeju, South Korea in 1997. She received the professional bachelor of the Early Childhood Education in February 2019 from Cheju Halla University, Jeju, South Korea and she has acquired the $2^{\circ}$ kindergarten instructor from Ministry of Science Education and Technology and the $2^{\circ}$ childcare provider from Ministry of Families and Social Welfare. She will be received the bachelor of the early childhood Jeju, South Korea. education in February 2020 from Cheju Halla University,

She has been working at the Jeju Youngrock Nursery since September 1, 2019. Currently, she is interested in the importance of educational methods over time and she wants to study which educational methods have a real educational effect. 